\title{
Espèces du genre Paramonostomum Lühe 1909 (fam. Notocotylidae Lühe 1909).
}

\author{
par Diane VAN STRYDONCK
}

Une petite collection de Notocotylidae d'Oiseaux a été recueillie en Belgique, elle comprend des espèces nouvelles du genre Paramonostomum Lühe 1909. Les déterminations ont été faites au Laboratoire de Zoologie (Vers) du Muséum National d'Histoire Naturelle, grâce à l'intervention du Centre National de la Recherche Scientifique.

Nous exprimons ici toute notre gratitude à M. R.-Ph. Dollfus et M. A.-G. Chabaud pour leur aide effective pendant ce travail. Nous tenons à remercier également l'I.R.S.I.A. (Institut pour l'encouragement de la Recherche Scientifique dans l'Industrie et l'Agriculture - Belgique) pour son aide financière.

\section{PARAMONOSTOMUM DOLLFUSI n. sp.}

\section{Matériel étudié}

Une vingtaine de Trématodes a été trouvée à Adinkerke (de Panne-sur-Mer), dans l'intestin grêle d'un Chauna torquata (Oken 1816). L'oiseau originaire d'Amérique du Sud est mort après avoir été mis en quarantaine pendant trois mois.

L'origine des parasites est incertaine. Nous ne savons pas s'il s'agit d'une infestation naturelle ou d'une infestation de captivité.

\section{Description (voir fig. 1 a).}

Distome à corps allongé, aplati dorso-ventralement, mesurant 2,2-3,85 $\mathrm{mm}$ de longueur et $340-429 \mu$ de largeur au niveau de la poche du cirre, et 477-517 $\mu$ au niveau des testicules. Pas d'ornementation cuticulaire visible à la surface du corps. Ventouse orale terminale, peu développée et mesurant 80-88 $\mu$. Ventouse ventrale absente. 


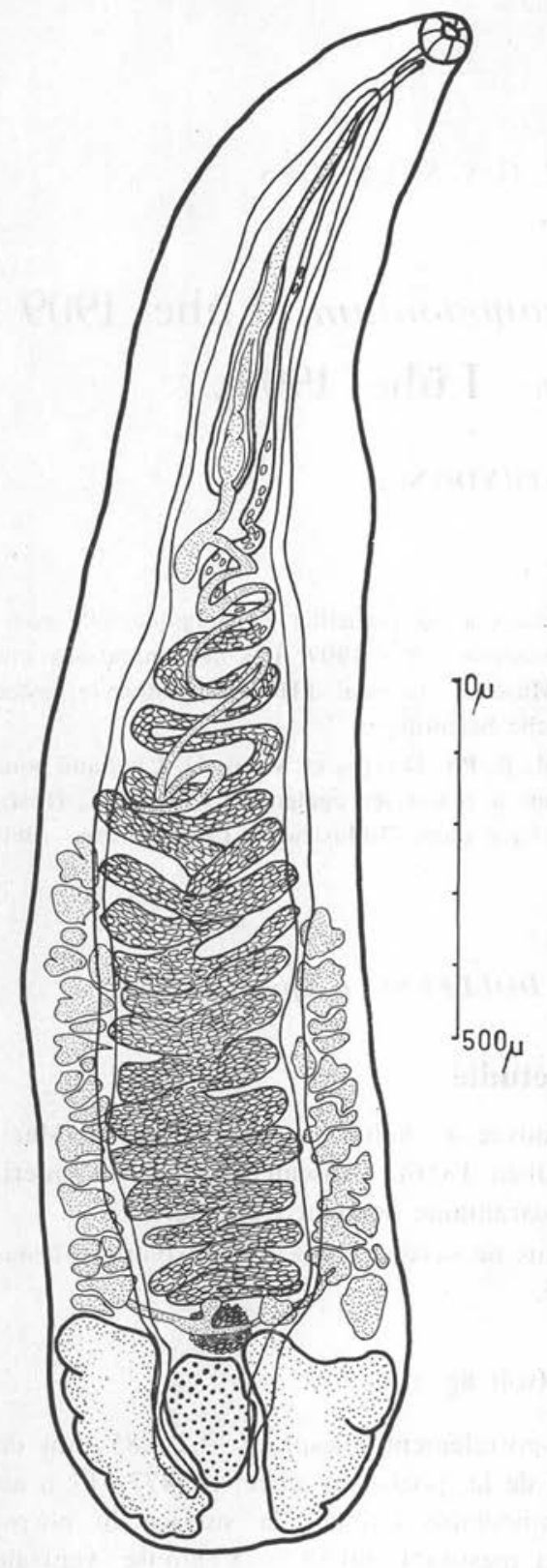

FIG. $1 a$

\section{Appareil digestif :}

Entonnoir buccal relativement court s'ouvrant dans l'œsophage. Pharynx absent.

Caeca longs, simples, cheminant de l'avant vers l'arrière, tout d'abord latéralement à l'utérus, puis entre l'utérus et les vitellogènes et enfin entre les testicules et l'ovaire.

\section{Appareil génital mâle :}

Testicules légèrement lobés, extracaecaux, situés en arrière des vitellogènes symétriquement par rapport à l'axe du corps et mesurant 283-381 $\mu$ longitudinalement et 157-233 $\mu$ transversalement.

Spermiductes visibles seulement à leur origine.

Poche du cirre disposée longitudinalement dans l'axe du corps, large de 65-70 $\mu$, longue de 655-1 $194 \mu$, s'étendant de la limite postérieure de la ventouse orale jusqu'au tiers de la longueur du corps (sa limite postérieure est située à 720-1 $290 \mu$ de l'extrémité antérieure du corps, soit environ aux 33-38/100 de la longueur totale).

Vésicule séminale interne, canal éjaculateur droit. Vésicule séminale externe visible. Cirre non visible sur nos préparations. 
Orifice génital situé en avant de la bifurcation intestinale; plusieurs dispositions ont été observées et sont figurées (voir fig. $1 \mathrm{~b}$ ).
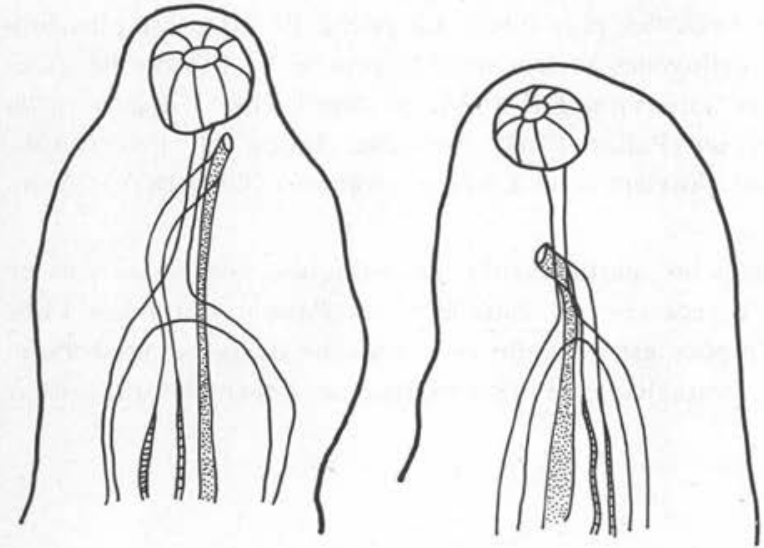

FIG. $1 b$

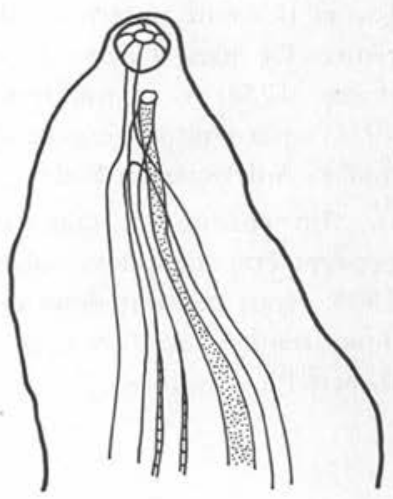

\section{Appareil génital femelle :}

Ovaire à peu près ellipsoïdal situé dans l'axe du corps entre les testicules et mesurant 190-228 $\mu$ longitudinalement et 96-123 $\mu$ transversalement.

Glande de Mehlis située en avant de l'ovaire dans l'axe du corps. Réceptacle séminal non observé,

Utérus s'étendant depuis l'ovaire jusqu'à la poche du cirre et accomplissant de larges méandres dont l'importance diminue de l'arrière vers l'avant.

Partie terminale de l'utérus longitudinale, parallèle à la poche du cirre dont l'extrémité (correspondant à peu près au tiers distal de la poche du cirre) plus musculeuse, est différenciée en metraterm.

Vitellogènes, composés d'un nombre variable de follicules, s'étendant latéralement depuis la limite postérieure de la vésicule séminale externe jusqu'à la limite antérieure des testicules.

Vitelloductes transverses, ayant pour origine les deux follicules postérieurs.

Eufs petits et ovales mesurant $16-20,9 \mu$ de longueur et 7,7-11,7 $\mu$ de largeur.

\section{Discussion.}

Les caractères précédents permettent de rattacher ces Trématodes aux Notocotylidae Lühe 1909, genre Paramonostomum Lühe 1909 (endoparasites à ventouse orale située à l'extrémité antérieure du corps et chez lesquels la ventouse ventrale, le pharynx et les groupes glandulaires de la face ventrale sont absents). 
Ils se distinguent des autres espèces connues dans ce genre par la position de l'orifice génital. L'espèce la plus proche est Paramonostomum casarcum Lal 1936. Cependant, la ventouse orale, l'ovaire et les testicules de Paramonostomum casarcum Lal 1936 sont plus grands et les testicules plus lobés. La poche du cirre est plus longue et la limite antérieure des vitellogènes se trouve plus proche de l'extrémité antérieure. De plus, Paramonostomum casarcum Lal 1936 est décrit chez Casarca rutila (Linné 1758) = Casarca serruginea Pallas 1764, Anatidae, Indes (cf. Peters J.-L. 1931) ; par contre, l'espèce décrite provient d'un Chauna torquata (Oken 1816), Anhimidae, Amérique du Sud.

En conclusion, étant données les particularités anatomiques, nos spécimens ne peuvent être identifiés à aucune espèce connue dans le genre Paramonostomum Lühe 1909. Nous pensons donc que l'espèce est nouvelle et proposons de la nommer Paramonostomum dollfusi n. sp. en témoignage de reconnaissance envers le Professeur Robert-Ph. Dollfus.

\section{PARAMONOSTOMUM CHABAUDI n. sp.}

\section{Matériel étudié}

Environ 100 Trématodes dans l'intestin grêle d'un huîtrier pie, Haematopus ostralegus Linné 1758 (Haematopodidae) et d'un canard colvert, Anas platyrhynchos Linné 1758 (Anatidae) morts à Knokke-sur-Mer).

\section{Description (voir fig. 2).}

Corps allongé et aplati dorso-ventralement mesurant 1,72-2,47 $\mathrm{mm}$ de longueur et 544-775 $\mu$ de largeur. Pas d'ornementation cuticulaire visible.

Ventouse orale terminale mesurant 100-128 $\mu$ de diamètre. Acétabulum absent.

\section{Appareil digestif :}

Entonnoir buccal s'ouvrant dans l'œsophage. Pharynx absent.

Caeca longs simples cheminant vers l'arrière du corps, latéralement à l'utérus passant ensuite entre les vitellogènes et l'utérus, puis entre l'ovaire et les testicules et atteignant le bord postérieur de l'ovaire.

\section{Appareil génital mâle :}

Testicules lobés, plus ou moins triangulaires, extérieurs aux caeca, symétriques 
par rapport à l'axe du corps, en arrière des vitellogènes et mesurant 176-345 $\mu$ longitudinalement et 143-250 $\mu$ transversalement.

Spermiductes invisibles sur les préparations.

Poche du cirre volumineuse disposée longitudinalement dans l'axe du corps, large de 70-80 $\mu$, longue de 447-732 $\mu$, s'étendant de la bifurcation intestinale jusqu'aux $2 / 5^{\circ}$ de la longueur du corps (limite postérieure située de 690 à $1017 \mu$ de l'extrémité antérieure du corps, soit environ aux 37-45/100 de la longueur totale). Tiens postérieur de la poche du cirre occupé par la vésicule séminale interne. En arrière de la poche du cirre, vésicule séminale externe visible. Orifice génital situé au niveau ou juste en arrière de la bifurcation intestinale.

\section{Appareil génital femelle :}

Ovaire de forme variable, présentant une échancrure sur son bord postérieur situé dans l'axe du corps, en arrière de l'utérus et mesurant 138-170 $\mu$ de longueur et $85-122 \mu$ de largeur.

Réceptacle séminal non observé.

Glande de Mehlis sphérique, située en avant de l'ovaire.

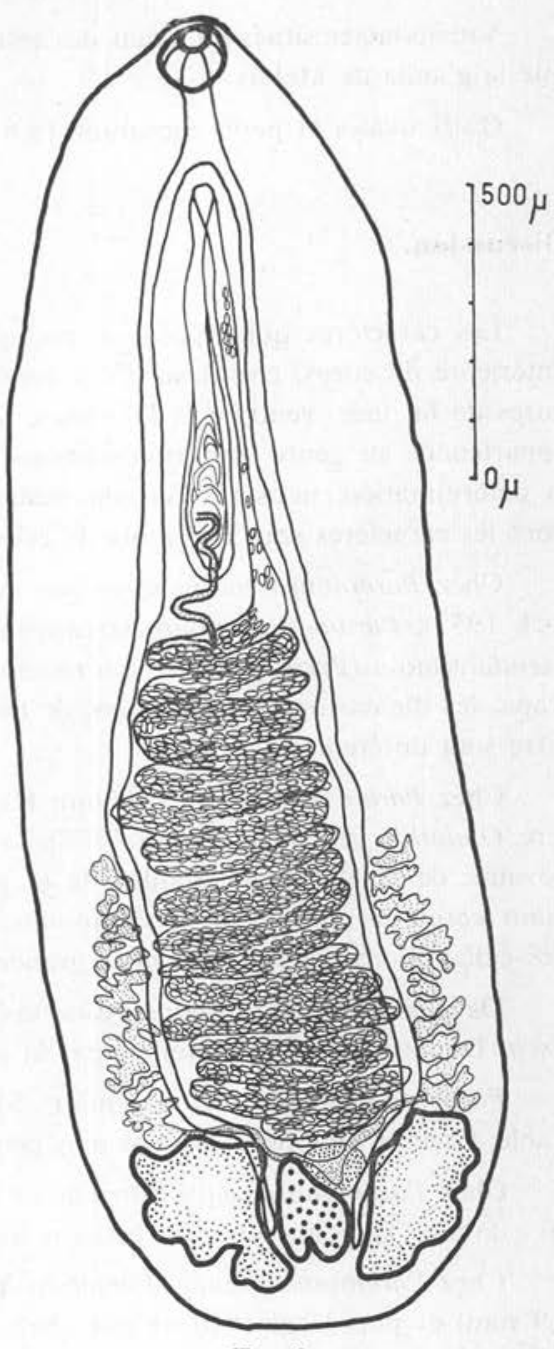

FIG. 2

Utérus s'étendant de l'ovaire jusqu'à la poche du cirre, accomplissant de larges boucles, dont l'importance diminue de l'arrière vers l'avant; partie terminale de l'utérus longitudinale, parallèle à la poche du cirre dont l'extrémité (correspondant à peu près à la demi-longueur de la poche du cirre) plus musculeuse, est différenciée en metraterm.

Vitellogènes situés le long des parois latérales; limite antérieure située aux 57-63/100 de la longueur du corps, limite postérieure juste en avant des testicules soit aux $82-86 / 100$ de la longueur totale. 
Vitelloductes situés en avant des testicules, réservoir vitellin situé au même niveau que la g'ande de Mehlis.

Eufs ovales et petits mesurant $18,8-25 \mu$ sur 9,5-15 $\mu$.

\section{Discussion.}

Les caractères qui précèdent (endoparasites à ventouse orale située à l'extrémité antérieure du corps, chez lesquels la ventouse ventrale, le pharynx, les groupes glandulaires de la face ventrale et le disque d'épines sont absents) indiquent une espèce appartenant au genre Paramonostomum Lühe 1909, Notocotylidae Lühe 1909. Pour la détermination, nous éliminerons successivement les espèces connues dans ce genre dont les caractères sont différents de ceux de nos spécimens.

Chez Paramonostomum alveatum (Mehlis 1846), Paramonostomum brantae (Bullock 1952), Paramonostomum parvum (Stunkard et Denihue 1931), Paramonostomum pseudalveatum (Price 1931) et Paramonostomum ionorne Travassos 1921, le corps est trapu, les dimensions des testicules, de l'ovaire, de la glande de Mehlis, de la poche du cirre sont différentes.

Chez Paramonostomum echinum Harrah 1922, seulement décrit chez un mammifère Ondathra zibethica (Linné 1758), la forme et les dimensions des vitellogènes, de l'ovaire, de la glande de Mehlis, de la poche du cirre sont différentes. Paramonostomum casarcum Lal 1936 et Paramonostomum querquedulum Lal 1936 diffèrent de nos échantillons par la forme et la grandeur de la poche du cirre.

De plus Paramonostomum casarcum Lal 1936, ainsi que Paramonostomum malerischi Dunagan 1957 en diffèrent par la position de l'orifice génital.

Paramonostomun microstomum Moghe 1932 et Paramonostomum actiditis Cable, Connor, Balling 1960 sont plus petits.

Chez Paramonostomum histrionici Ching 1961, les caeca présentent des diverticules; de plus, la poche du cirre est plus longue que chez nos spécimens.

Chez Paramonostomum elongatum Yamaguti 1934, le corps est plus long (jusqu'à $6,9 \mathrm{~mm})$ et plus large $(810 \mu)$ que chez notre spécimen; la poche du cirre atteint le milieu du corps.

Chez Paramonostomum ovatus Hsü 1935 les dimensions du corps et des ventouses sont supérieures à celles de nos exemplaires, de plus le corps est trapu.

Notre espèce se rapproche beaucoup de Paramonostomum bucephalae Yamaguti 1935, parasite de Bucephala clangula (Linné 1758), mais d'une part les dimensions du corps de l'ovaire et des testicules sont supérieures. D'autre part, chez Paramonostomum bucephalae Yamaguti 1935, la poche du cirre atteint les 50/100 de la longueur $\mathrm{du}$ corps et les vitellogènes remontent jusqu'à 50-55/100 de la longeur totale alors que chez nos échantillons, la poche du cirre n'atteint jamais la mi-longueur du corps 
(elle est située seulement dans le tiers antérieur) et la limite antérieure des vitellogènes est comprise entre les 55 et les $66 / 100$ de la longueur du corps.

Les caractères morphologiques de nos échantillons étant différents de ceux des espèces connues dans le genre Paramonostomum Lühe 1909, nous pensons qu'il s'agit d'une espèce nouvelle que nous nommerons Paramonostomum chabaudi n. sp. en témoignage de reconnaissance envers le Professeur A. G. Chabaud.

\section{Résumé}

Deux espèces du genre Paramonostomum Lühe 1909 sont décrites et discutées ; elles sont des espèces nouvelles.

- Paramonostomum dollfusi n. sp. de l'intestin grêle de Chauna torquata (Oken 1816) Anhimidae, se distingue par la place de l'orifice génital.

- Paramonostomum chabaudi n. sp. de l'intestin grêle de Haematopus ostralegus Linné 1758 et d'Anas platyrhynchos Linné 1758. Ce trématode se distingue par le développement de la poche du cirre et des vitellogènes.

\section{Bibliographie}

Caballero C. E., 1942. - Descripcion de un Paramonostomum (Trematoda: Notocotylidae) encontrado en los patos silvestres del lago de Texcoco V (1).Ann. Inst. Biol., 13 (1), pp. 91-95.

Cable, Connor, Balling, 1960. - Digenetic Trematodes of Puerto Rican shore Birds. Scient. Surv. Porto-Rico and Virgin Islands, V. 17 (12), pp. 189-255.

ChING H. L., 1961. - Three Trematodes from the harlequin Duck, Canad. Jn. of Zöol., 39 (3), pp. 373-376.

DAWES B., 1956. - The Trematoda with special reference to British and other European forms, Cambridge Univ. Press, 644 p.

Dunagan T., 1957. - Paramonostomum malerischi n. sp. (Trematoda, Digenea, Notocotylidae) from the emperor Goose (Philacte canagica L.) in Alaska. Jn. of Paras, 43, pp. 586-589.

Hsu Y. C., 1935. - Trematodes of fowls in Soöchow, Peking Nat. Hist. Bull., V, 10 (2), p. 141.

Kuc T., 1938. - New Trematodes from Chinese Birds. Peking Nat. Hist. Bull., V, 13 (2), pp. $129-136$.

Peters J. L., 1931. - Check-list of Birds of the World I, II, Harvard Univ. Press Cambridge, Massachusetts. 
Rothschild M., 1941. - Note on life histories of the genus Paramonostomum, Lühe, 1909, (Trematoda: Notocotylidae) with special reference to the excretory vesicle. Jn. of Paras., 27 (4), pp. 363-365.

SKRJABIN K. I., 1953. - Trematodes of Animals and Man V, VIII, Moskva.

Yamaguti S., 1934. - Studies on the helminth fauna of Japan. Part 3 Avian Trematodes II, Jap. Jn. Zoöl., V, 5 (4), pp. 543-583.

Yamaguti S., 1935. - Studies on the Helminth fauna of Japan. Part 5: Trematodes of Birds. III. Jap. Jn. of Zoöl., V, 6 (2), pp. 159-182.

Yamaguti S., 1958. - Systema Helminthum Vol. I Part I, II. The digenetic Trematodes of Vertebrates, Interscience Publishers Inc., New-York and London, pp. 1-1443.

(Laboratoire de Parasitologie - Ecole Vétérinaire, Gand - Belgique) 